\title{
Connecting the Dots: A Rare Cause of Pulmonary Nodules in a 13-Year-Old Boy
}

\section{Introduction}

$\mathrm{T}$ HE CAUSES OF multiple pulmonary nodules in a pediatric patient include infections, malignancies, sarcoidosis, granulomatosis with polyangiitis (GPA; formerly Wegener's granulomatosis), eosinophilic granulomatosis with polyangiitis (EGPA; formerly Churg Strauss), hypersensitivity pneumonitis, and lymphomatoid granulomatosis. The clinician is faced with the challenging task of combining clinical, radiologic, immunologic, and histopathologic data in order to distinguish several rare disorders with overlapping features.

\section{Case Report}

A 13-year-old male presented with a 6-month history of dry cough and a 1 week history of worsening shortness of breath and pleuritic chest pain. In addition, he had headaches, dry eyes/mouth (Sicca syndrome), and intermittent proximal interphalangeal joint swelling. There was no fever, night sweats, weight loss, skin rash, vision changes, or gastrointestinal symptoms. The family kept a dog and cat as pets, but there was no contact with other animals, construction sites, or cigarette smoke. There was a strong family history of rheumatologic disorders, including scleroderma and dermatomyositis.

The patient's vital signs were normal, and his weight was at the 63rd percentile. The physical exam was unremarkable except for mild epigastric tenderness. Initial blood tests, including blood count with differential, C-reactive protein, lactate dehydrogenase, uric acid, ferritin, electrolytes, renal and liver function, and urinalysis, were normal. The sedimentation rate was slightly elevated at $18 \mathrm{~mm} / \mathrm{h}$ (reference range 0 $15 \mathrm{~mm} / \mathrm{h}$ ). A chest X-ray demonstrated nodular opacities (Fig. $1 \mathrm{~A}$ and $\mathrm{B}$ ); chest computed tomography (CT) confirmed multiple (>40) small, solid, bilateral pulmonary nodules and no lymphadenopathy (Fig. 1C-F). Most nodules were pleural based, and some had central lucency. Serum angiotensinconverting enzyme level was $32 \mathrm{IU} / \mathrm{L}$ (reference range 16-65 IU/L). Rheumatologic workup, including antinuclear antibody, anti-double-stranded DNA antibody, antineutrophil cytoplasmic antibody (ANCA), antimyeloperoxidase, and von Willebrand factor antigen, was negative. Fungal serology panel, aspergillus antigen, urine histoplasma antigen, tuberculin purified protein derivative skin test, quantiferon $\mathrm{TB}$, and mycoplasma and chlamydia serologies were negative. He had normal spirometry and plethysmographic lung volumes but a diffusion capacity of $70 \%$ predicted. A thoracoscopic wedge resection of two nodules demonstrated exuberant granulomatous inflammation involving the lung and pleura. A mixture of necrotizing and non-necrotizing epithelioid granulomas was present (Fig. 2). Granulomas were noted surrounding airways and vessels and focally invaded small vessel walls, breaking into the lumen (Fig. 3). Special stains for organisms performed on multiple sections, including Ziehl-Neelson, Fite, Grocott's methenamine silver, and Warthin-Starry, were all negative. No foreign material was identified. Bacterial and fungal cultures were negative.

With negative microbiological serology studies, tissue stains, and cultures, infectious causes were ruled out. The presence of vasculitis and extrapulmonary manifestations raised the possibility of a small vessel vasculitis, but the absence of uncontrolled asthma, chronic rhinosinusitis, peripheral eosinophilia, renal disease, or positive ANCA ruled out GPA and EGPA. The clinical, radiologic, laboratory, and histologic findings in the patient were not typical for classic sarcoidosis. The presence of granulomatous vasculitis and necrosis and exclusion of other entities in the differential diagnosis were consistent with necrotizing sarcoid granulomatosis (NSG). NSG is a rare condition characterized by sarcoid-like granulomas, variable degrees of necrosis, vasculitis, frequent extrapulmonary manifestations, and a generally favorable prognosis. ${ }^{1}$

The patient continued to have headaches, joint pain, mouth/eye dryness, and chest pain. A head computed tomography (CT)/magnetic resonance imaging (MRI)/magnetic resonance angiogram (MRA) confirmed no cerebral or vascular abnormalities. Given previous case series identifying favorable outcomes in patients with NSG treated with steroids, ${ }^{2,3}$ the patient received pulse therapy of $1,000 \mathrm{mg}$ intravenous methylprednisolone daily for 3 days, and was subsequently transitioned to oral corticosteroid therapy with prednisone, $60 \mathrm{mg}$ daily. Within 1 month of starting steroids, he experienced symptomatic relief of headaches, dry mouth, and joint pain, as well as normalization of his diffusion capacity. During the sixth week of his steroid wean, a repeat chest CT demonstrated near complete resolution of the

Divisions of ${ }^{1}$ Pediatric Pulmonology and ${ }^{2}$ Pediatric Rheumatology, Department of Pediatrics and Communicable Diseases, and ${ }^{3}$ Department of Pathology, University of Michigan Medical School, Ann Arbor, Michigan. 

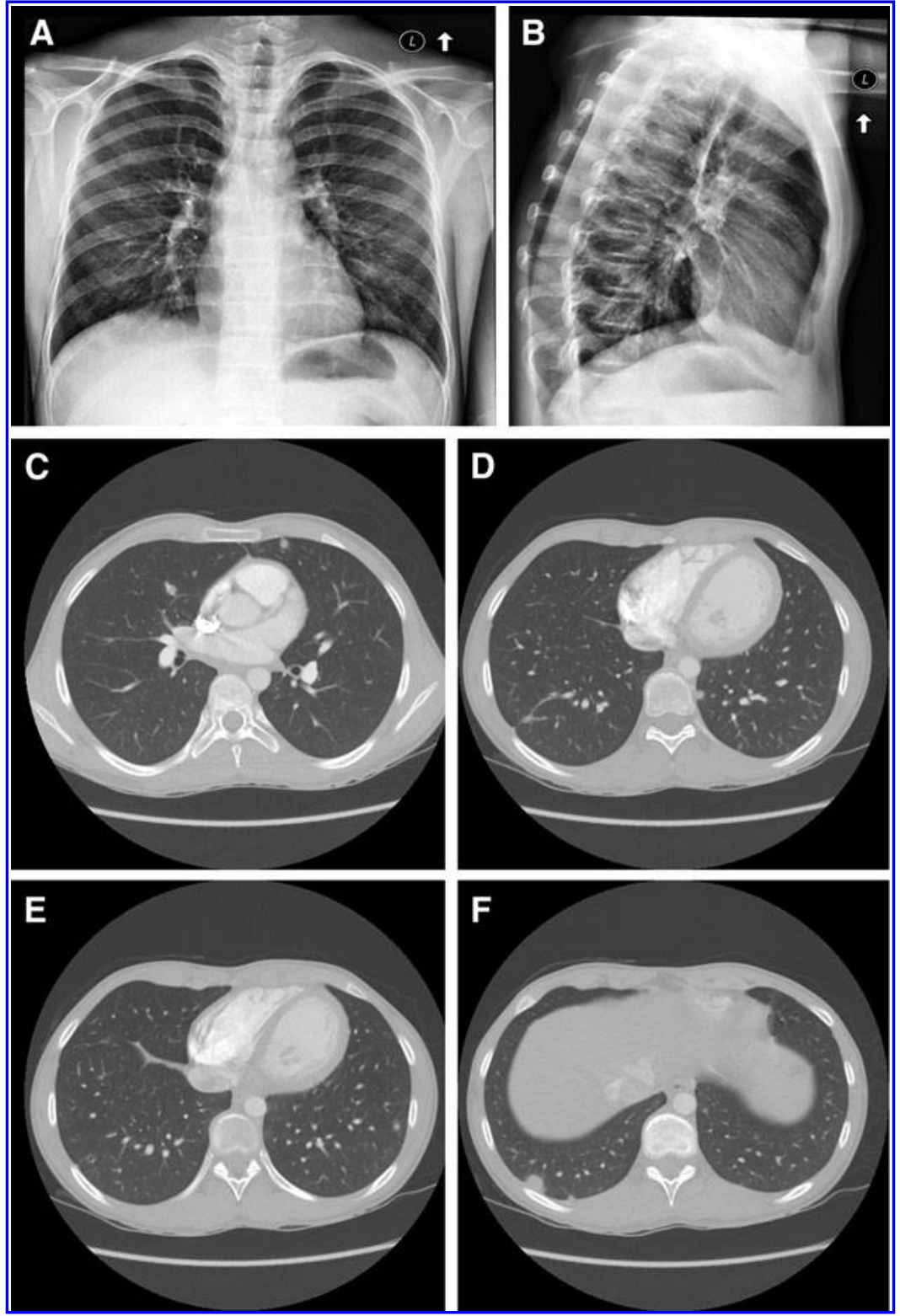

FIG. 1. (A) and (B) Antero-posterior and lateral chest $\mathrm{X}$-rays demonstrate nodular opacities. (C)-(F) Chest computed tomography $(\mathrm{CT})$ scan with contrast using $2.5 \mathrm{~mm}$ cuts. Nodules are mostly pleural based. One right lower lobe nodule has a central lucency $(\mathbf{E})$. nodules, and the sedimentation rate had normalized. Chest pain resolved, although the patient complained of abdominal pain, as well as tenderness in the proximal interphalangeal joints and sacroiliac joint. A pelvic MRI identified irregularity and sclerosis of the right sacroiliac joint consistent with history of sacroiliitis but no active synovial inflammation. He was treated with azathioprine (50 mg daily) as a steroidsparing agent, and experienced resolution of the arthritis. However, his course was complicated by persistent generalized myalgias and frequent headaches consistent with amplified musculoskeletal pain syndrome and nonsteroidal anti-inflammatory drug (NSAID) overuse.

\section{Discussion}

Necrotizing sarcoid granulomatosis was described for the first time in 1973 by Liebow who identified 11 patients with sarcoid-like granulomas, variable degrees of necrosis, and vasculitis. ${ }^{1}$ Since then, nearly 100 adult cases and 10 pediatric cases have been reported in the literature. ${ }^{2-11}$ Extra- pulmonary involvement is estimated to occur in nearly $86 \%$ of adult patients, and has been reported in half of the pediatric cases reported. ${ }^{2,3}$ The majority of patients experience resolution of their disease, and some require steroids or immunosuppression, particularly those with more severe systemic or neurologic manifestations.

Necrotizing granulomas have a broad differential, which includes mycobacteria, fungal infections, GPA, and foreign body-type inflammation. Non-necrotizing granulomas are more often a manifestation of a noninfectious cause such as sarcoidosis and vasculitides. The pathology of NSG is unique in that it demonstrates features of both sarcoidosis and granulomatous vasculitides, raising the question of whether this disease represents a variation of sarcoidosis with necrosis of the granuloma and vessels, or a necrotizing angiitis with sarcoid reaction. The cell types involved, microbiological stains, and associated patterns of vasculitis and necrosis provide useful clues into the underlying cause.

NSG shares several pulmonary and extrapulmonary clinical features with sarcoidosis, including a generally 


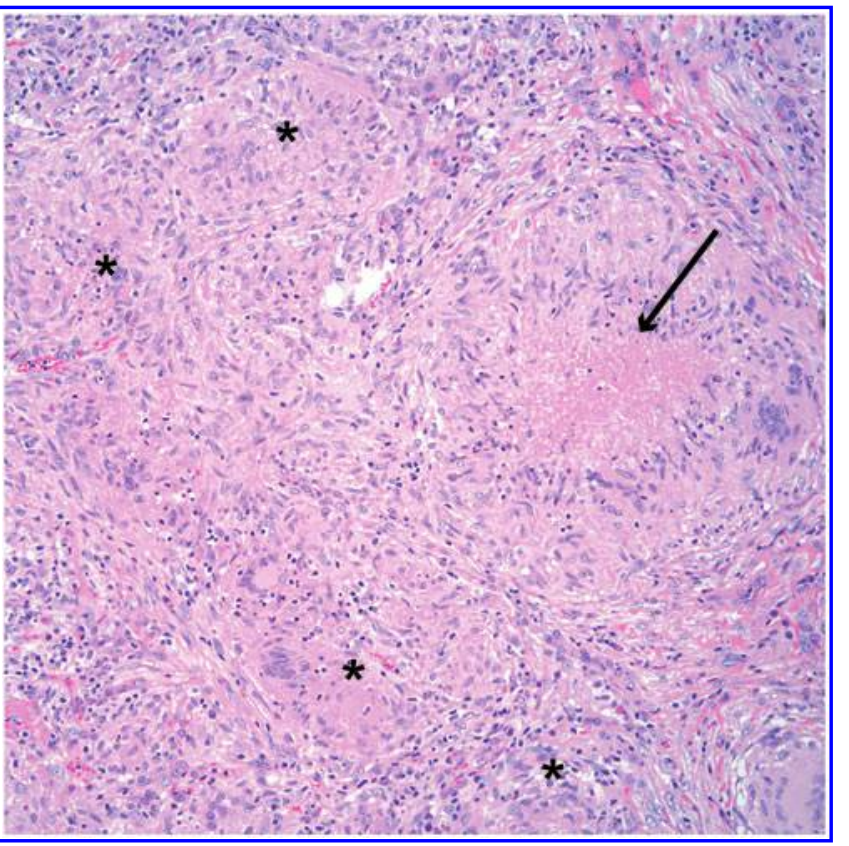

FIG. 2. Multiple small epithelioid non-necrotizing granulomas composed of lymphocytes, epithelioid histiocytes, and scattered multinucleated giant cells are seen (stars) in addition to one granuloma with central coagulative necrosis (arrow). Color images available online at www.liebertpub $. \mathrm{com} / \mathrm{ped}$

favorable prognosis ${ }^{2,3,12}$ and similar immunopathologic ${ }^{7}$ and histopathologic findings. ${ }^{13-15}$ In fact, an estimated 5\% of granulomas in sarcoidosis show the typical histology of NSG, including necrotizing granulomas. ${ }^{15,16}$ Lazzarini et al. reported a 15-year-old girl with NSG and a strong family

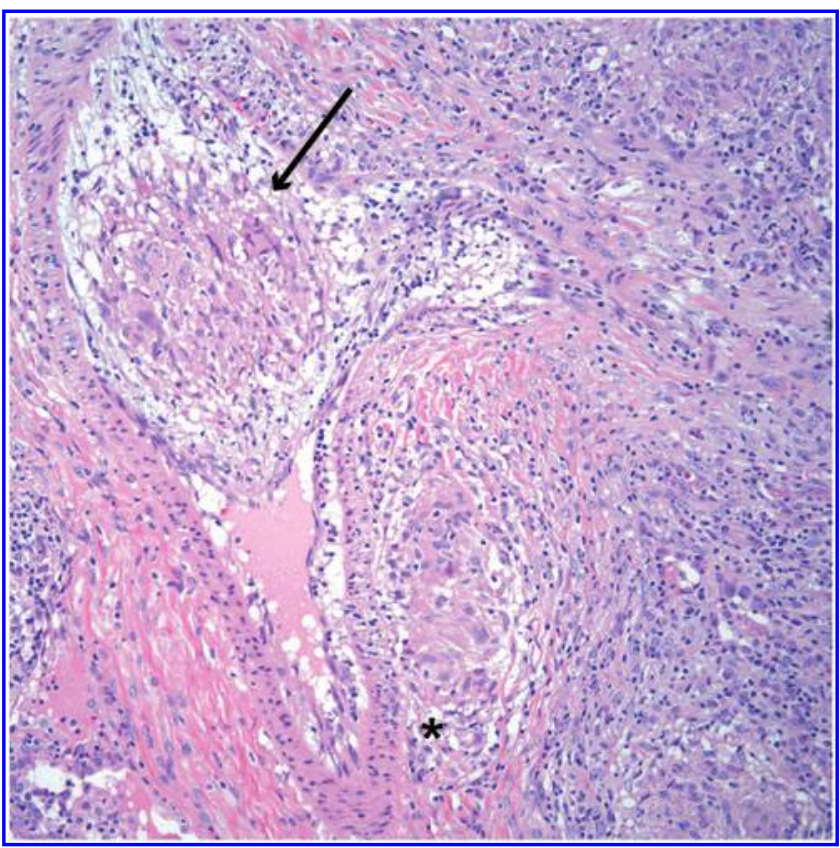

FIG. 3. One epithelioid granuloma is present adjacent to a small vessel (star), and one granuloma invaded the vascular wall and broke into the lumen (arrow). Color images available online at www.liebertpub.com/ped history of sarcoidosis, and suggested a possible autoimmune link between the two diseases. ${ }^{8}$ However, the peripheral location of the nodules, lack of hilar adenopathy, and normal angiotensin converting enzyme would suggest that NSG and sarcoidosis are distinct entities. NSG shares some pathologic features with granulomatous angiitides, but is not associated with an elevated ANCA, renal disease, or asthma.

The differential diagnosis of pulmonary granulomatous diseases in children is broad and requires a thorough evaluation for hematologic, infectious, immune-mediated, and vasculitic disorders. To our knowledge, this patient is the eleventh pediatric case of NSG reported in the literature. NSG is an example of a disease with unclear classification, as some of its features overlap with other granulomatous and vasculitic disorders.

\section{Author Disclosure Statement}

No competing financial interests exist.

\section{References}

1. Liebow AA. The J. Burns Amberson lecture-pulmonary angiitis and granulomatosis. Am Rev Respir Dis 1973; 108:1-18.

2. Quaden C, Tillie-Leblond I, Delobbe A, Delaunois L, Verstraeten A, et al. Necrotising sarcoid granulomatosis: clinical, functional, endoscopical and radiographical evaluations. Eur Respir J 2005;26:778-785.

3. Chittock DR, Joseph MG, Paterson NA, McFadden RG. Necrotizing sarcoid granulomatosis with pleural involvement. Clinical and radiographic features. Chest 1994;106: 672-676.

4. Aliberti S, Falleni M, Tarsia P, Vago G, Zerbi P, et al. A 13-year-old female with shortness of breath and pleuritic chest pain. Eur Respir J 2006;28:876-882.

5. Beach RC, Corrin B, Scopes JW, Graham E. Necrotizing sarcoid granulomatosis with neurologic lesions in a child. J Pediatr 1980;97:950-953.

6. Fernandes SR, Singsen BH, Hoffman GS. Sarcoidosis and systemic vasculitis. Semin Arthritis Rheum 2000;30: 33-46.

7. Heinrich D, Gordjani N, Trusen A, Marx A, Hebestreit H. Necrotizing sarcoid granulomatosis: a rarity in childhood. Pediatr Pulmonol 2003;35:407-411.

8. Lazzarini LC, de Fatima do Amparo Teixeira M, Souza Rodrigues R, Marcos Nunes Valiante P. Necrotizing sarcoid granulomatosis in a family of patients with sarcoidosis reinforces the association between both entities. Respiration 2008;76:356-360.

9. Panigada S, Ullmann N, Sacco O, Gambini C, Bush A, Rossi GA. Necrotizing sarcoid granulomatosis of the lung in a 12-year-old boy with an atypical clinical course. Pediatr Pulmonol 2012;47:831-835.

10. Singh N, Cole S, Krause PJ, Conway M, Garcia L. Necrotizing sarcoid granulomatosis with extrapulmonary involvement. Clinical, pathologic, ultrastructural, and immunologic features. Am Rev Respir Dis 1981;124:189192.

11. Tauber E, Wojnarowski C, Horcher E, Dekan G, Frischer T. Necrotizing sarcoid granulomatosis in a 14-yr-old female. Eur Respir J 1999;13:703-705.

12. Sweiss NJ, Patterson K, Sawaqed R, Jabbar U, Korsten P, et al. Rheumatologic manifestations of sarcoidosis. Semin Respir Crit Care Med 2010;31:463-473. 
13. Popper HH, Klemen H, Colby TV, Churg A. Necrotizing sarcoid granulomatosis-is it different from nodular sarcoidosis? Pneumologie 2003;57:268-271.

14. Churg A, Carrington CB, Gupta R. Necrotizing sarcoid granulomatosis. Chest 1979;76:406-413.

15. Churg A. Pulmonary angiitis and granulomatosis revisited. Hum Pathol 1983;14:868-883.

16. Rosen Y, Moon S, Huang CT, Gourin A, Lyons HA. Granulomatous pulmonary angiitis in sarcoidosis. Arch Pathol Lab Med 1977;101:170-174.
Address correspondence to: Thomas G. Saba, MD

Department of Pediatrics and Communicable Diseases University of Michigan Medical School Ann Arbor, MI 48109

E-mail: tsaba@umich.edu

Received for publication June 20, 2014; accepted after revision August 2, 2014. 